\title{
Localization of a spin-orbit-coupled Bose-Einstein condensate in a bichromatic optical lattice
}

\author{
Yongshan Cheng,,$^{1, *}$ Gaohui Tang, ${ }^{1, \dagger}$ and S. K. Adhikari ${ }^{2, \ddagger}$ \\ ${ }^{1}$ Department of Physics, Hubei Normal University, Huangshi 435002, People's Republic of China \\ ${ }^{2}$ Instituto de Física Teórica, Universidade Estadual Paulista, 01.140-070 São Paulo, São Paulo, Brazil
}

(Received 7 March 2014; published 3 June 2014)

\begin{abstract}
We study the localization of a noninteracting and weakly interacting Bose-Einstein condensate (BEC) with spin-orbit coupling loaded in a quasiperiodic bichromatic optical lattice potential using the numerical solution and variational approximation of a binary mean-field Gross-Pitaevskii equation with two pseudospin components. We confirm the existence of the stationary localized states in the presence of the spin-orbit and Rabi couplings for an equal distribution of atoms in the two components. We find that the interaction between the spin-orbit and Rabi couplings favors the localization or delocalization of the BEC depending on the phase difference between the components. We also studied the oscillation dynamics of the localized states for an initial population imbalance between the two components.
\end{abstract}

DOI: 10.1103/PhysRevA.89.063602 PACS number(s): 03.75.Mn, 03.75.Kk, 71.70.Ej, 72.15.Rn

\section{INTRODUCTION}

The first experimental observation of spin-orbit (SO) coupling in Bose-Einstein condensates (BECs) [1] has stimulated widespread experimental and theoretical discussions in different fields. Spin-orbit-coupled cold atoms represent a fascinating and fast developing area of research and lead to rich physical effects [2]. In ultracold atomic systems, a variety of synthetic SO coupling can be engineered by two counterpropagating Raman lasers that couple two hyperfine ground states, and most experimental parameters can be controlled at will by optical or magnetic means [3]. Using this technique, the SO coupling has been created in the atomic Fermi [4] and Bose gases [1,5]. Motivated by these experimental breakthroughs, a great number of theoretical activities have been devoted to the SO-coupled BEC, including superfluidity [6], vortex structure [7], and the soliton [8] of a SO-coupled BEC. There have also been extensive theoretical efforts toward understanding the physics of the SO-coupled Fermi gases [9]. A generic binary mean-field Gross-Pitaevskii (GP) equation is derived in Ref. [10] which provide the starting point for the theoretical study of many-body dynamics in SO-coupled BECs. Similar models have also been derived in other studies [11] and have been employed in investigating the localized modes [12] and other topics of SO-coupled BECs $[13,14]$.

Another topic of current interest is the localization of a BEC in a disorder potential. Since Anderson predicted the localization of a noninteracting electron wave in solids with a disorder potential about 50 years ago [15], localization phenomena have been studied in different types of waves, including atomic matter waves. Two experimental groups reported the localization of a noninteracting BEC in two different kinds of one-dimensional (1D) disordered potentials. Billy et al. [16] observed the exponential tail of the spatial density distribution of a ${ }^{87} \mathrm{Rb}$ BEC after releasing it into a 1D waveguide with a controlled disorder potential created by

\footnotetext{
*yong_shan@163.com

${ }^{\dagger} \operatorname{tgh} 19900201 @ 163 . c o m$

adhikari@ift.unesp.br; http://www.ift.unesp.br/users/adhikari
}

a laser speckle. Roati et al. [17] observed the localization of a noninteracting ${ }^{39} \mathrm{~K}$ BEC in a $1 \mathrm{D}$ quasiperiodic bichromatic optical lattice (OL) potential. A bichromatic OL is realized by a primary lattice perturbed by a weak secondary lattice with incommensurate wavelength [18]. The experimental realizations of three-dimensional (3D) localization of a spinpolarized Fermi gas of ${ }^{40} \mathrm{~K}$ [19] atoms and a BEC of ${ }^{87} \mathrm{Rb}$ [20] atoms in a $3 \mathrm{D}$ speckle potential were also reported. Much theoretical work has been done on Anderson localization of a BEC [21]. Recently, some theoretical investigations have been reported for the localization of a SO-coupled particle moving in a $1 \mathrm{D}$ quasiperiodic potential [22] and random potential [23].

In this paper, we investigate the statics and dynamics of localization of a noninteracting and weakly interacting BEC with Rabi and SO couplings, trapped in a 1D quasiperiodic bichromatic OL potential, similar to the one used in the experiment of Roati et al. [17], using a mean-field GP equation with two pseudospin components. The localized states are stationary for an equal occupation in the pseudospin states. But when there is a population imbalance, spontaneous oscillation between the two pseudospin components of the localized state takes place. We restrict ourselves to a study of the effect of the phase difference between the components and of the SO and Rabi couplings on the statics and dynamics of localization. Within a range of parameters, most of the atoms can be localized in a single OL site, the density profiles of the localized BEC are quite similar to a Gaussian shape, and the variational approximation can be used for some analytical understanding of the localized states [24]. In view of the SO coupling, the initial ansatz of the wave function we choose in our analysis has a somewhat more complicated form in order to get an understanding of the characteristics of the localized BECs. The stability criterion of the stationary localized states is discussed by performing a standard linear stability analysis. We also study the tails of the localized states, where we focus on the spatially extended nature of wave functions with exponential decay corresponding to a weak Anderson localization [15,25]. We also study the dynamics of atom transfer between the two localized components with a population imbalance.

In Sec. II we present a brief account of the coupled mean-field model and the bichromatic OL potential used in 
the study. The analytical expressions for the atom transfer ratio and phase difference between the two localized states and the width of the two localized states are obtained by variational analysis of the mean-field model. Various aspects of stationary localization are studied by variational approximation and numerical solution of the mean-field equation. In particular, the effects of the phase difference between components and of the SO and Rabi couplings on the localization of the BEC are investigated in Sec. III. Some dynamics of the nonstationary localized states are presented in Sec. IV. A brief summary and future perspective are given in Sec. V.

\section{ANALYTICAL CONSIDERATION}

In the electronic states of an atom the SO coupling naturally appears due to the magnetic energy associated with this coupling because of the electronic charge. In the case of neutral atoms engineering with electromagnetic fields is required for the SO coupling to contribute to the BEC. To create a simple SO coupling in the laboratory, Lin et al. [1] consider two internal spin states of ${ }^{87} \mathrm{Rb}$ hyperfine state $5 S_{1 / 2}$ : $|\uparrow\rangle=\mid F=$ $\left.1, m_{F}=0\right\rangle$ and $|\downarrow\rangle=\left|F=1, m_{F}=-1\right\rangle$, where $F$ and $m_{F}$ are the total angular momentum of the hyperfine state and its $z$ projection. These states are called pseudo-spin-up and pseudospin-down states in analogy with the two spin components of a spin-1/2 particle. The SO coupling between these states is then realized with strength $\Omega$ using two counterpropagating Raman lasers, and this SO coupling is equivalent to that of an electronic system with equal contributions from Rashba [26] and Dresselhaus [27] couplings and with an external uniform magnetic field. We consider a BEC with internal up and down pseudospin states $|\uparrow\rangle$ and $|\downarrow\rangle$ confined in a spin-independent quasi-1D potential $V(x)$ oriented in the longitudinal $(x)$ direction. A strong harmonic potential of angular frequency $\omega_{\perp}$ is applied in the transverse $y, z$ directions, and the transverse dynamics of the condensate is assumed to be frozen to the respective ground states of the harmonic traps. Then, the single-particle quasi-1D Hamiltonian of the system under the action of a strong transverse trap of angular frequency $\omega_{\perp}$ in the $y-z$ plane can be written as $[1,12,13]$

$$
H_{0}=\frac{p_{x}^{2}}{2 m}+\frac{\hbar k_{L}}{m} p_{x} \sigma_{z}+\frac{\hbar \Omega}{2} \sigma_{x}+V(x),
$$

where $p_{x}=-i \hbar \partial_{x}$ is the momentum operator along the $x$ direction, $m$ is the mass of an atom, $\sigma_{x, z}$ are the usual $2 \times 2$ Pauli matrices, $k_{L}$ is the wave number of the Raman lasers that couple the two atomic hyperfine states, and the coupling strength $\Omega$ is the Rabi frequency acting as a Zeeman field. If the interactions among the atoms in the BEC are taken into account, in the Hartree approximation, the dynamics of the BEC of $N$ atoms can be described by the $2 \times 2$ nonlinear $1 \mathrm{D}$ GP equation $[8,10,11]$ :

$$
i \hbar \frac{\partial \psi}{\partial t}=H_{0} \psi+G \psi
$$

where $\psi=\left(\psi_{1}, \psi_{2}\right)^{T}$ is the two-component mean-field wave function with normalization $\int d x\left(\left|\psi_{1}\right|^{2}+\left|\psi_{2}\right|^{2}\right)=N$. The two time-dependent spinor wave functions $\psi_{j}(j=1,2)$ describe the two pesudospin components $(|\uparrow\rangle$ and $|\downarrow\rangle)$ of the
BEC. The nonlinear term has the $2 \times 2$ matrix form [10,28]

$$
G=\left(\begin{array}{cc}
\frac{2 \hbar^{2} a\left|\psi_{1}\right|^{2}+2 \hbar^{2} a_{12}\left|\psi_{2}\right|^{2}}{m a_{\perp}^{2}} & 0 \\
0 & \frac{2 \hbar^{2} a\left|\psi_{2}\right|^{2}+2 \hbar^{2} a_{12}\left|\psi_{1}\right|^{2} \mid}{m a_{\perp}^{2}}
\end{array}\right),
$$

where, to make the parameters of the model tractable, we take the two intraspecies scattering lengths $a_{j}$ to be equal, $a_{1}=a_{2}=a$, and where $a_{12}$ is the interspecies scattering length and $a_{\perp}=\sqrt{\hbar /\left(m \omega_{\perp}\right)}$ is the harmonic oscillator length of the transverse trap. In actual experiments it is possible to control these scattering lengths independently by optical [29] and magnetic [30] Feshbach resonance techniques. In dimensionless units the coupled GP equations for the wave function $u_{j} \equiv u_{j}(x, t)=\psi_{j}(x, t) \sqrt{a_{\perp}}(j=1,2)$ can be written as [12]

$$
\begin{aligned}
i \frac{\partial u_{j}}{\partial t}= & -\frac{1}{2} \frac{\partial^{2} u_{j}}{\partial x^{2}}+i(-1)^{j} \gamma \frac{\partial u_{j}}{\partial x}+\Gamma u_{(3-j)} \\
& +\left(g\left|u_{j}\right|^{2}+g_{12}\left|u_{(3-j)}\right|^{2}\right) u_{j}+V(x) u_{j}
\end{aligned}
$$

where the spatial variable $x$, time $t$, density $\left|u_{j}\right|^{2}$, and energy are expressed in normalized units $a_{\perp}, \omega_{\perp}^{-1}, a_{\perp}^{-1}$, and $\hbar \omega_{\perp}$, respectively. The interaction nonlinearities are [28] $g=2 a / a_{\perp}^{2}, g_{12}=2 a_{12} / a_{\perp}^{2}$, the SO-coupling strength is $\gamma \equiv$ $k_{L} a_{\perp}$, and the Rabi-coupling strength is $\Gamma \equiv \Omega /\left(2 \omega_{\perp}\right)$. The normalization $\int_{-\infty}^{\infty}\left|u_{j}\right|^{2} d x=N_{j}$, where $N_{j}$ is the number of atoms in component $j$. As in the experiment of Roati et al. [17], the bichromatic OL potential $V(x)$ is taken as the linear combination of two polarized standing-wave OL potentials of incommensurate wavelengths:

$$
V(x)=\sum_{l=1}^{2} A_{l} \sin ^{2}\left(k_{l} x\right),
$$

with $A_{l}=2 \pi^{2} s_{l} / \lambda_{l}^{2}(l=1,2)$, where $\lambda_{l}$ are the wavelengths of the OL potentials, $s_{l}$ are their intensities, and $k_{l}=2 \pi / \lambda_{l}$ are the corresponding wave numbers. In this investigation, the irrational ratio between the two OLs is set to be [21] $k_{2} / k_{1}=(\sqrt{5}-1) / 2$, the inverse of the golden ratio. In the actual experiment of Roati et al. [17], the parameter was set as $k_{2} / k_{1}=1.1972$. Without losing generality, we further take $\lambda_{1}=10$ and $s_{1}=10, s_{2}=0.3 s_{1}$, which are roughly the same parameters as in the experiment of Roati et al. [17].

The dynamics of the BEC can be investigated by the Gaussian variational approach [24]. This approach is justified for small contact repulsion and for small SO coupling when the localized state has a spatial extension over a single OL site. In such a situation, the central density of the localized state has an approximately Gaussian shape. However, at large distances the localized state has a long exponential tail. The Gaussian variational approach can describe the Anderson localization experiment of Ref. [17] in the noninteracting regime. In this approach, the Lagrangian density for Eq. (4) is

$$
\begin{aligned}
\mathcal{L}= & \sum_{j=1}^{2}\left\{\frac{i}{2}\left(u_{j}^{*} \dot{u}_{j}-u_{j} \dot{u}_{j}^{*}\right)-(-1)^{j} \frac{i}{2} \gamma\left[u_{j}^{*} u_{j}^{\prime}-u_{j}\left(u_{j}^{*}\right)^{\prime}\right]\right. \\
& \left.-\frac{1}{2}\left|u_{j}^{\prime}\right|^{2}-\frac{1}{2} g\left|u_{j}\right|^{4}-V(x)\left|u_{j}\right|^{2}\right\}-g_{12}\left|u_{1}\right|^{2}\left|u_{2}\right|^{2} \\
& -\Gamma\left(u_{1}^{*} u_{2}+u_{1} u_{2}^{*}\right),
\end{aligned}
$$


where the asterisk denotes the complex conjugate, the prime denotes $d / d x$, and the overdot denotes $d / d t$. The Gaussian ansatz with the time-dependent variational parameters $N_{j}, w_{j}, \beta_{j}$, and $\phi_{j}$ is used to study the dynamics:

$u_{j}(x, t)=\frac{1}{\pi^{1 / 4}} \sqrt{\frac{N_{j}}{w_{j}}} \exp \left[-\frac{x^{2}}{2 w_{j}^{2}}+(-1)^{j} i \beta_{j} x+i \phi_{j}\right]$,

where $N_{j}$ and $w_{j}$ represent the number of atoms and width of the BEC and $\beta_{j}$ and $\phi_{j}$ are chirp and phase. The time dependence of these variables is not explicitly shown in the following. The effective Lagrangian of the system (4) is found by substituting Eq. (7) into Eq. (6) and integrating over space variables [24]:

$$
\begin{aligned}
L= & \sum_{j=1}^{2} N_{j}\left\{-\dot{\phi}_{j}+\gamma \beta_{j}-\frac{1}{2}\left(\frac{1}{2 w_{j}^{2}}+\beta_{j}^{2}+\frac{g N_{j}}{\sqrt{2 \pi} w_{j}}\right)\right. \\
& \left.+\frac{1}{2} \sum_{l=1}^{2} A_{l}\left[\exp \left(-k_{l}^{2} w_{j}^{2}\right)-1\right]\right\}-\frac{g_{12} N_{1} N_{2}}{\sqrt{\pi\left(w_{1}^{2}+w_{2}^{2}\right)}} \\
& -2 \Gamma \cos \left(\phi_{2}-\phi_{1}\right) \sqrt{N_{1} N_{2}} L_{\Gamma},
\end{aligned}
$$

where

$$
L_{\Gamma}=\sqrt{\frac{2 w_{1} w_{2}}{w_{1}^{2}+w_{2}^{2}}} \exp \left[-\frac{\left(\beta_{1}+\beta_{2}\right)^{2} w_{1}^{2} w_{2}^{2}}{2\left(w_{1}^{2}+w_{2}^{2}\right)}\right] .
$$

We further define the atom transfer ratio between the two localized states, $R=\left(N_{2}-N_{1}\right) / N$, and the phase difference, $\phi \equiv \phi_{2}-\phi_{1}$. Using the Euler-Lagrange equation

$$
\frac{\partial L}{\partial \alpha}-\frac{d}{d t} \frac{\partial L}{\partial \dot{\alpha}}=0
$$

where $\alpha$ denotes the variational parameters $\phi_{j}, N_{j}, w_{j}$, and $\beta_{j}$, we obtain the following equations:

$$
\begin{aligned}
\dot{\phi}= & \gamma\left(\beta_{2}-\beta_{1}\right)-\frac{1}{4}\left(\frac{1}{w_{2}^{2}}-\frac{1}{w_{1}^{2}}\right) \\
& -\frac{g N}{2 \sqrt{2 \pi}}\left(\frac{1+R}{w_{2}}-\frac{1-R}{w_{1}}\right)-\frac{1}{2}\left(\beta_{2}^{2}-\beta_{1}^{2}\right) \\
& +\frac{1}{2} \sum_{l=1}^{2} A_{l}\left[\exp \left(-k_{l}^{2} w_{2}^{2}\right)-\exp \left(-k_{l}^{2} w_{1}^{2}\right)\right] \\
& +\frac{g_{12} R N}{\sqrt{\pi\left(w_{1}^{2}+w_{2}^{2}\right)}}+\frac{2 R \Gamma \cos \phi}{\sqrt{1-R^{2}}} L_{\Gamma} \equiv G(R, \phi), \\
0= & \frac{1}{2 w_{j}^{3}}+\frac{g N_{j}}{2 \sqrt{2 \pi} w_{j}^{2}}-w_{j} \sum_{l=1} A_{l} k_{l}^{2} \exp \left(-k_{l}^{2} w_{j}^{2}\right) \\
& +\frac{g_{12} N_{3-j} w_{j}}{\sqrt{\pi\left(w_{1}^{2}+w_{2}^{2}\right)^{3}}}-2 \Gamma \cos \phi \sqrt{\frac{N_{3-j}}{N_{j}}} \frac{\partial L_{\Gamma}}{\partial w_{j}}, \\
\dot{R}= & -2 \Gamma \sin \phi \sqrt{1-R^{2}} L_{\Gamma} \equiv F(R, \phi), \\
0= & \gamma-\beta_{j}+2 \Gamma \cos \phi \sqrt{\frac{N_{3-j}}{N_{j}}} \frac{\left(\beta_{2}+\beta_{1}\right) w_{1}^{2} w_{2}^{2}}{w_{1}^{2}+w_{2}^{2}} L_{\Gamma} .
\end{aligned}
$$

Equation (13) shows that the transfer ratio $R$ is explicitly dependent on $\Gamma$, which implies that the Rabi coupling leads to atom transfer between the two localized states.

\section{STATIONARY LOCALIZED STATE}

The stationary states are obtained by setting the time derivative in Eqs. (11) and (13) to zero. If $R=0$, i.e., $N_{1}=N_{2}$, we obtain $\beta_{1}=\beta_{2} \equiv \beta$ from Eq. (14), $w_{1}=w_{2} \equiv w$ from Eq. (12), and $\dot{\phi}=0$ from Eq. (11). Consequently, the two localized states given by Eq. (7) are identical. Hence, the simple stationary solutions, in this case, are

$$
\begin{array}{ll}
R=0, \quad \phi=0 \quad \text { (in phase), } \\
R=0, \quad \phi=\pi \quad \text { (out of phase). }
\end{array}
$$

Hence, Eqs. (12) and (14) can be rewritten as

$$
\begin{aligned}
0= & \frac{1}{2 w^{3}}+N \frac{g+g_{12}}{4 \sqrt{2 \pi} w^{2}}-w \sum_{l=1}^{2} A_{l} k_{l}^{2} \exp \left(-k_{l}^{2} w^{2}\right) \\
& \pm 2 \Gamma w \beta^{2} \exp \left(-\beta^{2} w^{2}\right), \\
0= & \gamma-\beta \pm 2 \Gamma w^{2} \beta \exp \left(-\beta^{2} w^{2}\right),
\end{aligned}
$$

where a plus corresponds to $\phi=0$ and a minus corresponds to $\phi=\pi$. We find that the stationary states are related to the external trapping potential, nonlinearity, phase difference, and SO and Rabi couplings. In order to focus our attention on the effects of the phase difference and SO and Rabi couplings on the localization of the BEC, here, we will restrict ourselves first to the noninteracting regime. The weakly interacting regime, and even the noninteracting one, could be achieved by reducing the $s$-wave scattering length by means of Feshbach resonances [30]. We take $g=g_{12}=0$ with potential (5) in the following investigations.

We solve Eq. (4) by the real- or imaginary-time split-step Fourier spectral method with a space step of 0.04 and a time step of 0.001 . In real-time propagation, to obtain the stationary localized states, we take the stationary solution of Eq. (4) for $g=g_{12}=\Gamma=0$ and $V(x)=x^{2} / 2$, e.g., $u_{j}(x)=\pi^{-1 / 4} \exp \left[-x^{2} / 2+(-1)^{j} i \gamma x+i \phi_{j 0}\right]$, as the initial input. Successively, the parabolic trap is slowly turned off, the bichromatic OL is slowly turned on, and the parameter $\Gamma$ is added gradually in steps of 0.000001 from zero to the final value. To investigate the effects of the phase difference, we take $\phi_{10}=\phi_{20}=0$ for the in-phase case and $\phi_{10}=0$ and $\phi_{20}=\pi$ for the out-of-phase configuration in the initial input pulses.

In this section for the calculation of stationary states we take throughout $N_{1}=N_{2}=1$. Let us first investigate the effects of the coefficient $\gamma$ and $\Gamma$ on the noninteracting localized states when $\gamma \times \Gamma=0$ and $g=g_{12}=0$. If $\gamma=0$, a solution of Eq. (18) is $\beta=0$. Then, the last term on the right-hand side of Eq. (17) is zero, so that the widths are not related to the phase difference and Rabi coupling $\Gamma$. Also, if $\Gamma=0$, Eq. (17) shows that the widths are independent of the phase difference $\phi$ and SO coupling $\gamma$. In both cases the widths are determined by only the external trapping potential and nonlinearity. In these cases, the variational width is 0.9688 , and the numerical width $\left(w^{2}=2 \int_{-\infty}^{+\infty} x^{2}|u|^{2} d x\right)$ is 0.9945 . The numerical simulation of Eq. (4) shows that $u_{1}$ and $u_{2}$ are similar: $\left|u_{1}\right|^{2}=\left|u_{2}\right|^{2}$, but 

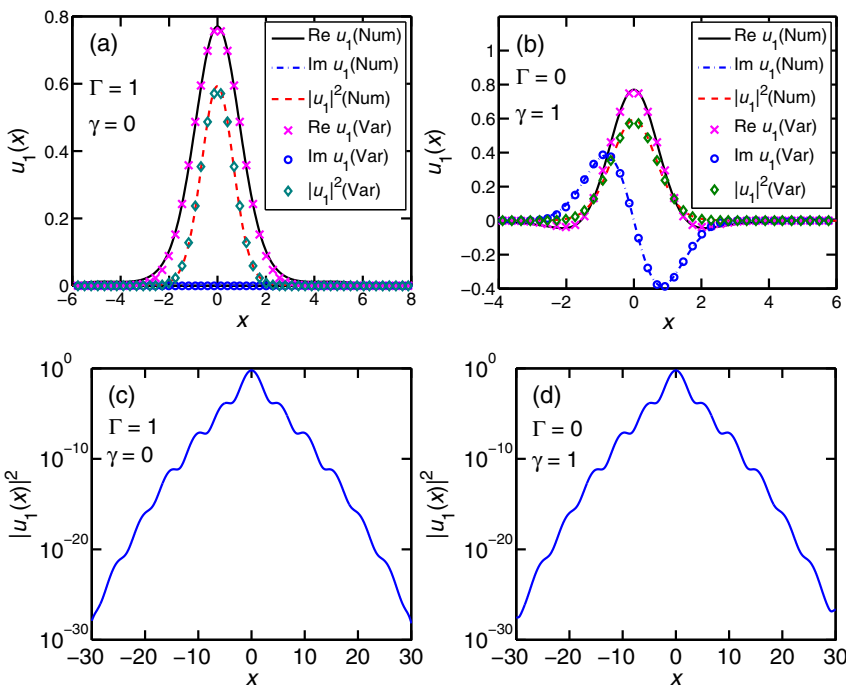

FIG. 1. (Color online) Real (Re) and imaginary (Im) parts of the numerical (Num) and variational (Var) wave function $u_{1}$ ( $x$ ) of Eq. (4) with the bichromatic OL potential (5) and the corresponding density $\left|u_{1}(x)\right|^{2}$ vs $x$ for $g=g_{12}=0$ and (a) $\Gamma=1, \gamma=0, N_{1}=N_{2}=1$ and (b) $\Gamma=0, \gamma=1$. The corresponding numerical densities $\left|u_{1}(x)\right|^{2}$ in $\log$ scale are shown in (c) and (d). Because of the similarity between $u_{1}$ and $u_{2}$, only the wave function $u_{1}$ is plotted. All quantities are dimensionless. (The same applies to the following figures.)

$\operatorname{Im}\left(u_{1}\right)=-\operatorname{Im}\left(u_{2}\right)$ and $\operatorname{Re}\left(u_{1}\right)=\operatorname{Re}\left(u_{2}\right)$ for $\phi_{10}=\phi_{20}=0$, and $\operatorname{Im}\left(u_{1}\right)=\operatorname{Im}\left(u_{2}\right)$ and $\operatorname{Re}\left(u_{1}\right)=-\operatorname{Re}\left(u_{2}\right)$ for $\phi_{10}=0$ and $\phi_{20}=\pi$. Because of the similarity between $u_{1}$ and $u_{2}$, we plot only $u_{1}$ hereafter, as in Figs. 1(a) and 1(b), which illustrate the variational and numerical results for the localized states. The variational wave function is obtained by solving Eqs. (17) and substituting $w$ and $\beta$ into Eq. (7). We find that the variational results are in good agreement with the numerical results.

Anderson localization in a weakly disordered potential is characterized by a long exponential tail of the localized state $[15,25]$. To observe the effects of the SO and Rabi couplings on the tail region, we plot in Figs. 1(c) and 1(d) the density distribution $\left|u_{1}\right|^{2}$ of the stationary BEC in log scale. The parameters $\gamma$ and $\Gamma$ have no effect on the exponential tail when $\gamma \times \Gamma=0$, as confirmed by the numerical simulation of Eq. (4) with different $\gamma$ and $\Gamma$.

Next, we consider $\gamma \times \Gamma \neq 0$. Because of the interaction between $\gamma$ and $\Gamma$, now the width of the stationary state should depend on the phase difference and SO and Rabi couplings. However, Eqs. (17) and (18) show that the width of the localized state is determined by the difference $(\gamma-\beta)$.

In the case of $\phi=0$ (in phase), the last term in Eq. (17) is positive for positive Rabi coupling $\Gamma$ and contributes to a delocalization of the BEC as the positive kinetic-energy term (the first term on the right-hand side) and the positive repulsive-interaction term (the second term on the right-hand side). The only term contributing to localization is the negative bichromatic OL term (the third term on the right-hand side) in Eq. (17). Hence a large positive $\Gamma$ should lead to a partially delocalized state occupying a large spatial region extending over several OL sites. Such a localized state over multiple OL sites has a multihump structure. To acquire a single-hump
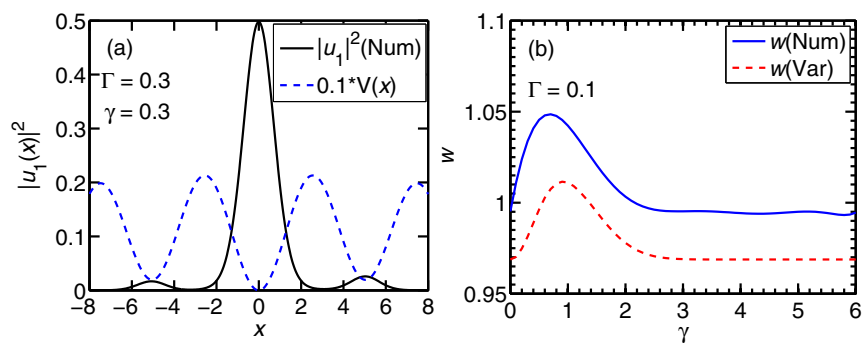

FIG. 2. (Color online) Numerical and variational results for $\phi=$ 0 (in phase), $g=g_{12}=0, N_{1}=N_{2}=1$ (a) for density $\left|u_{1}\right|^{2}$ vs $x$ for $\gamma=\Gamma=0.3$ and (b) for width vs $\gamma$ for $\Gamma=0.1$. To compare, the OL potential $0.1 V(x)$ is also plotted in (a).

localized state, Rabi coupling $\Gamma$ must be small. Then, it follows from Eq. (18) that if $\gamma$ is large enough, $\beta \simeq \gamma$, and the width is independent of $\beta, \gamma$, and $\Gamma$. For example, we obtain $\beta=3.5, w=0.9688$ by numerically solving Eqs. (17) and (18) with $\gamma=3.5, \Gamma=0.1$. The numerical integration of Eqs. (4) and (5) shows that a single-humped localized state splits into a multihumped state occupying more than one OL site with the increase of $\Gamma$, as illustrated in Fig. 2(a) for $\gamma=\Gamma=0.3$. If $\Gamma=0.1$ is small, the density profile occupies only one OL site within a wide range of parameter $\gamma$. In Fig. 2(b) we compare the numerical and variational widths of the localized state for $\Gamma=0.1$ and different $\gamma$ and find that the width is the smallest for $\gamma=0$, implying that a positive $\gamma$ contributes to a slight delocalization for small $\gamma$. For larger $\gamma$ ( $>3$ ), the width is practically independent of $\gamma$. These findings are consistent with variational Eqs. (17) and (18). In Fig. 2(b) the numerical width is slightly larger than the variational width, consistent with a long exponential tail of the former. We also studied the tails of the density profiles in logarithmic scale and found that the effect of $\gamma$ on the tails is small.

In the case of $\phi=\pi$ (out of phase), from Eq. (17) we find that a positive $\Gamma$ favors localization and the set of Eqs. (17) and (18) has a real solution for width $w$ with arbitrary positive $\Gamma$ and $\gamma$. In this case the density profile of the stable localized state could be modulated with a non-Gaussian shape even within the single OL site, as illustrated in Fig. 3(a). From a numerical solution of Eq. (4), we obtain the phase diagram of Fig. 3(b) of $\gamma$ and $\Gamma$, showing the regions where the density profile is Gaussian and non-Gaussian. To confirm this, a plot of the numerical and variational widths versus $\Gamma$ is presented in Fig. 3(c) for $\gamma=1$, which shows that a positive $\Gamma$ contributes a localization of the BEC because the width for $\Gamma>0$ is less than that for $\Gamma=0$. So far we considered the central density of the localized states. A careful examination of the densities of the localized states reveals that at large distances from the central region the localized states always have a long exponential tail within both Gaussian and non-Gaussian regimes. In fact this is the most important earmark of Anderson localization. A measure of this tail can be given by a localization length $L_{\text {loc }}$ obtained by fitting the density tail to the exponential function $\sim \exp \left(-|x| / L_{l o c}\right)[25,31]$. The localization effect of a positive $\Gamma$, however, will have a major influence on the exponential tail. For $\gamma=1$, the effect of $\Gamma$ on the localization length $L_{l o c}$ is presented in Fig. 3(c). As expected, the localization effect of a nonzero $\Gamma$ makes the localization length always be smaller than 

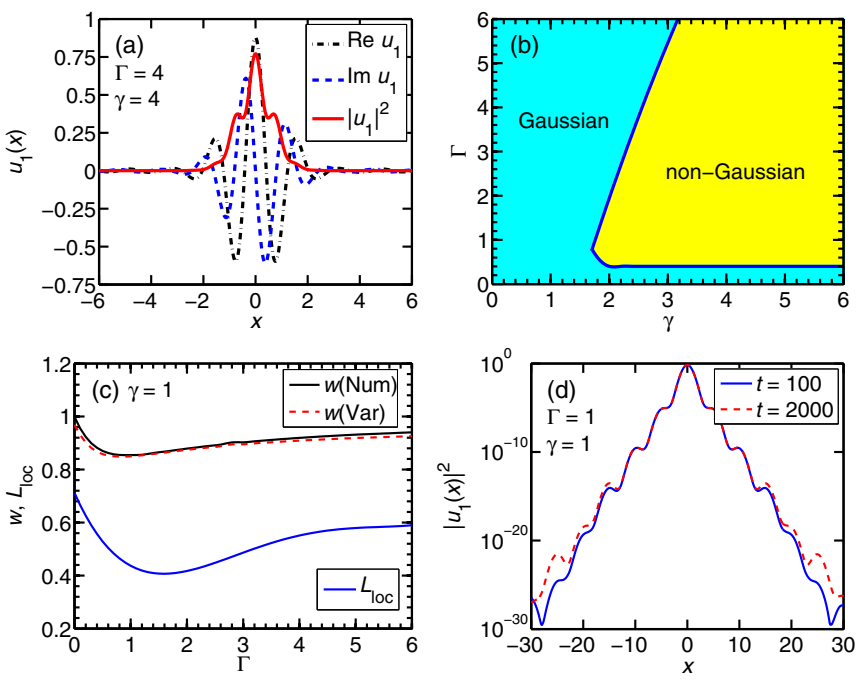

FIG. 3. (Color online) Numerical results for $\phi=\pi$ (out of phase), $g=g_{12}=0, N_{1}=N_{2}=1$ (a) for the wave function $u_{1}$ and density for $\gamma=\Gamma=4$, (b) for a $\Gamma-\gamma$ phase plot showing Gaussian and non-Gaussian localization regions, (c) for width and localization length for $\gamma=1$ and different $\Gamma$, and (d) for density at large times $t$ for $\gamma=\Gamma=1$. In (c) the variational results of widths are also shown.

that for $\Gamma=0$. Furthermore, at large time scales, Fig. 3(d) illustrates that a subdiffusion occurs below a certain critical $\Gamma \times$ $\gamma$, as analyzed numerically in Ref. [32], where a subdiffusion appears above a certain strength of nonlinearity. Nevertheless, spreading induced by the subdiffusion is rather slow [32]. So the localization lengths shown in Fig. 3(c) are meaningful.

Third, we consider the stability of the solutions (15) and (16) by performing a standard linear stability analysis. Introducing small fluctuations around the stationary solution $\left(R_{0}, \phi_{0}\right), R^{\prime}(t)=R(t)-R_{0}, \phi^{\prime}(t)=\phi(t)-\phi_{0}$, and linearizing Eqs. (11) and (13), a set of two linear equations is obtained:

$$
\begin{aligned}
& \frac{d R^{\prime}(t)}{d t}=F_{R}\left(R_{0}, \phi_{0}\right) R^{\prime}(t)+F_{\phi}\left(R_{0}, \phi_{0}\right) \phi^{\prime}(t), \\
& \frac{d \phi^{\prime}(t)}{d t}=G_{R}\left(R_{0}, \phi_{0}\right) R^{\prime}(t)+G_{\phi}\left(R_{0}, \phi_{0}\right) \phi^{\prime}(t),
\end{aligned}
$$

where the subscripts $R$ and $\phi$ denote a derivative with respect to the respective variable. Assuming the solution of $R^{\prime}(t)$ and $\phi^{\prime}(t)$ in exponential form, $\sim \exp (\zeta t)$, the eigenvalue $\zeta$ is given by

$$
\begin{aligned}
2 \zeta= & F_{R}\left(R_{0}, \phi_{0}\right)+G_{\phi}\left(R_{0}, \phi_{0}\right) \pm\left\{\left[F_{R}\left(R_{0}, \phi_{0}\right)\right.\right. \\
& \left.\left.-G_{\phi}\left(R_{0}, \phi_{0}\right)\right]^{2}+4 F_{\phi}\left(R_{0}, \phi_{0}\right) G_{R}\left(R_{0}, \phi_{0}\right)\right\}^{1 / 2} .
\end{aligned}
$$

From Eqs. (11) and (13), we find $F_{R}\left(R_{0}, \phi_{0}\right)=0, G_{\phi}\left(R_{0}, \phi_{0}\right)=$ 0 , and

$$
\begin{gathered}
F_{\phi}\left(R_{0}, \phi_{0}\right)=\mp 2 \Gamma \exp \left(-\beta^{2} w^{2}\right), \\
G_{R}\left(R_{0}, \phi_{0}\right)=N\left[\frac{g_{12}-g}{\sqrt{2 \pi} w} \pm \frac{2 \Gamma}{N} \exp \left(-\beta^{2} w^{2}\right)\right],
\end{gathered}
$$

which leads to the eigenvalues

$$
\zeta= \pm\left[F_{\phi}\left(R_{0}, \phi_{0}\right) G_{R}\left(R_{0}, \phi_{0}\right)\right]^{1 / 2} .
$$

If the eigenvalue $\zeta$ is purely imaginary, the stationary solutions denoted by Eqs. (15) and (16) are stable with respect to small perturbations. The constraints for stability are

$$
\begin{aligned}
& \frac{g_{12}-g}{\sqrt{2 \pi} w}+\frac{2 \Gamma}{N} \exp \left(-\beta^{2} w^{2}\right)>0 \quad(\phi=0), \\
& \frac{g_{12}-g}{\sqrt{2 \pi} w}-\frac{2 \Gamma}{N} \exp \left(-\beta^{2} w^{2}\right)<0 \quad(\phi=\pi) .
\end{aligned}
$$

A straightforward conclusion from Eqs. (25) and (26) is that any stationary state is stable for $g=g_{12}$. In addition, the conditions for stability are different for the in-phase and out-of-phase localized states when $g \neq g_{12}$.

Now, let us investigate the effect of the positive nonlinearity on the localized states in the presence of the SO and Rabi couplings. As shown by Eq. (17), the stable localized states can exist within a range of parameter $g, g_{12}, \Gamma$, and $\gamma$, and this has also been confirmed by the numerical integration of Eq. (4). The numerical and variational widths versus $g\left(=g_{12}\right)$ are plotted in Fig. 4(a) for $\phi=0, \gamma=\Gamma=0.1$ and in Fig. 4(b) for $\phi=\pi, \gamma=\Gamma=1$. With these parameters, the localized states are confined in a single OL site. Figures 4(a) and 4(b) indicate that the numerical and variational widths increase monotonically as the nonlinearity (increase of repulsion) increases. The numerical results are slightly larger than the variational results because of the exponential tail of the localized state. If the nonlinearity is large enough, however, the localized states develop undulating tails occupying more than one OL site and cannot be described well by the Gaussian ansatz (7). For $g \neq g_{12}$, the typical numerical and variational densities $\left|u_{1}\right|^{2}$ versus $x$ are illustrated in Fig. 4(c) for $\phi=0$ and in Fig. 4(d) for $\phi=\pi$. The parameters are chosen to meet the stability criteria (25) and (26). The stabilities for those localized states are tested by suddenly changing the OL's intensity $s_{1}$ from 10 to 9.5 and continually running the
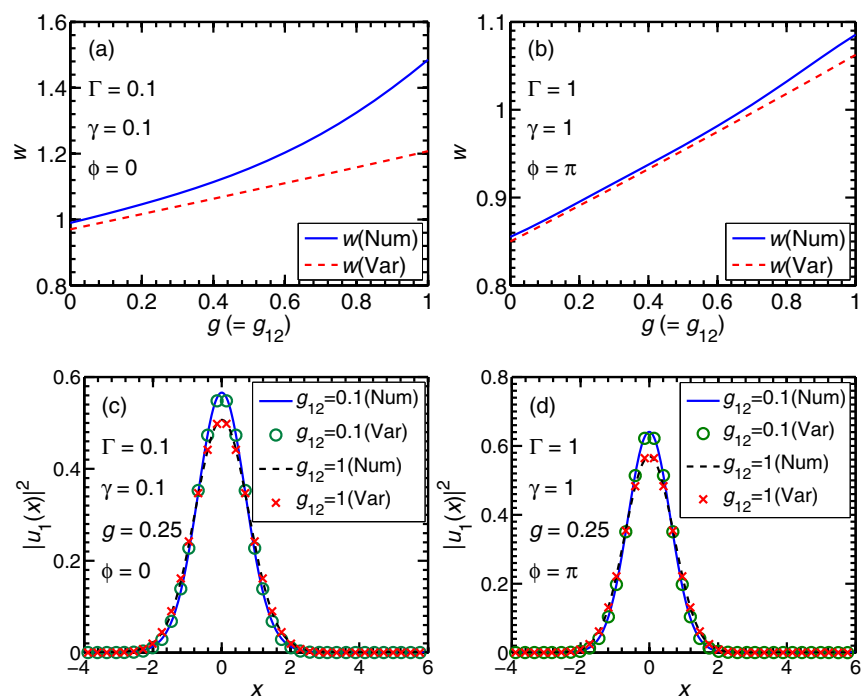

FIG. 4. (Color online) Numerical (Num) and variational (Var) widths vs $g\left(=g_{12}\right)$ for (a) $\Gamma=\gamma=0.1$ and $\phi=0$ and (b) $\Gamma=\gamma=1$ and $\phi=\pi$. Numerical and variational wave functions $\left|u_{1}\right|^{2}$ vs $x$ for $g \neq g_{12}$ and for (c) $\Gamma=\gamma=0.1, \phi=0$ and (d) $\Gamma=\gamma=1, \phi=\pi$. 
real-time program. The localized states are again found to be stable against the small perturbation.

\section{DYNAMICS OF THE LOCALIZED STATE}

For the stationary states studied so far one must have $N_{1}=$ $N_{2}$. A little imbalance between $N_{1}$ and $N_{2}$ leads to periodic atom transfer between two components. The localized states may exist in that case, although the wave functions change with time with periodic atom transfer between components.

In order to get further insight into the effects of the coefficient $\gamma$ and $\Gamma$ on the localized states, we now study some dynamics of the noninteracting and weakly interacting BECs. As shown by Eq. (13), Rabi-coupling strength $\Gamma$ plays an important role for the atom transfer between components. We investigate the effects of SO coupling $\gamma$ and Rabi coupling $\Gamma$ on the atom transfer ratio $R(t)$. If $\gamma=0$ and $g=g_{12}$, Eqs. (11) and (13) become

$$
\begin{gathered}
\dot{R}(t)=-2 \Gamma \sin \phi(t) \sqrt{1-R^{2}(t),} \\
\dot{\phi}(t)=\frac{2 \Gamma R(t) \cos \phi(t)}{\sqrt{1-R^{2}(t)}},
\end{gathered}
$$

with the solutions

$$
\begin{aligned}
R(t) & =A \cos (2 \Gamma t+B), \\
|\cos \phi(t)| & =\frac{\sqrt{1-A^{2}}}{\sqrt{1-R^{2}(t)}} \quad(R<1),
\end{aligned}
$$

where $A$ and $B$ are integration constants, which are determined by the initial values $R(0)$ and $\phi(0)$. Equation (29) shows that the period of atom transfer is determined solely by $\Gamma$. From Eqs. (29) and (30), we can deduce that the integration constants $A$ and $B$ change periodically with $\phi(0)$. The minimum of $A$ is $A=R(0)$, corresponding to $\phi(0)=0$, and the maximum is $A=1$, corresponding to $\phi(0)=\pi / 2$. Notice that, theoretically, Eqs. (28) and (30) have the singular points $R(t)= \pm 1$, which should be encountered if $\phi(0)=\pi / 2$. However, the numerical integration of Eq. (4) reveals that $R(t)= \pm 1$ can be achieved when $\phi(0)=\pi / 2$, and the atom transfer can go on in this case with $R(t)$ oscillating periodically between \pm 1 .

Contour plots of numerical density profiles versus time $t$ are displayed in Fig. 5(a) for $\left|u_{1}\right|^{2}$ and Fig. 5(b) for $\left|u_{2}\right|^{2}$ for $g=$ $g_{12}=0$. The periodic atom transfer between the components is clear in these plots. A quantitative measure of this oscillation is given by the plot of numerical and variational estimates of atom transfer ratio $R(t)$ versus $t$ in Fig. 5(c). The variational $R(t)$ is obtained by a numerical integration of Eqs. (27) and (28) with the fourth-order Runge-Kutta method. To obtain the numerical $R(t)$, we first obtain the stationary states employing the imaginary-time method solving Eqs. (4) and (5) with $\gamma=$ $\Gamma=0$ and $N_{j}(0)$ such that $R(0)=\left[N_{2}(0)-N_{1}(0)\right] /\left[N_{2}(0)+\right.$ $\left.N_{1}(0)\right]$ and $\phi(0)=\phi_{20}(0)-\phi_{10}(0)=0$. Successively, at $t=$ 0 , we employ the real-time propagation of Eq. (4) with the same parameters and just change $\Gamma$ from 0 to 1 . The atom transfer between components starts with a nonzero $\Gamma$, and a numerical $R(t)$ is obtained by calculating $R(t)=\left[N_{2}(t)-\right.$ $\left.N_{1}(t)\right] /\left[N_{2}(t)+N_{1}(t)\right]$ with $N_{j}(t)=\int_{-\infty}^{-\infty}\left|u_{j}(x, t)\right|^{2} d x$.

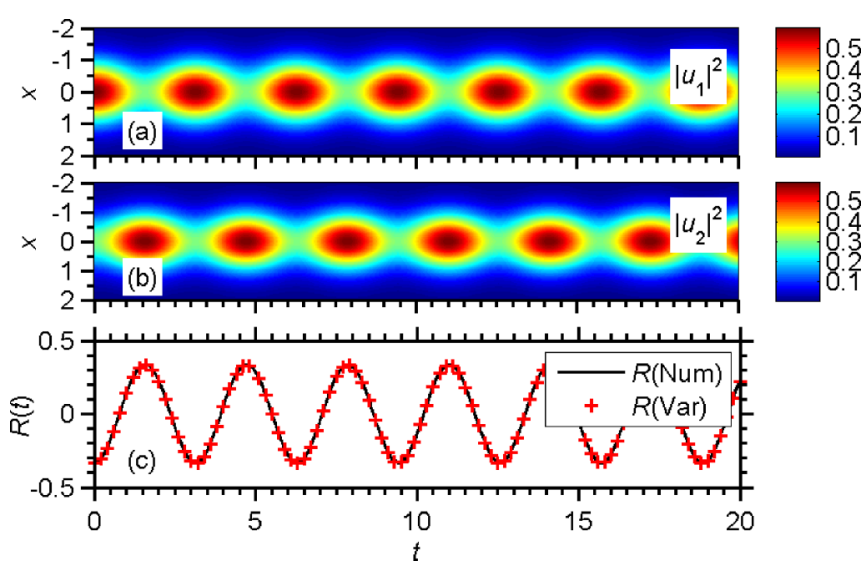

FIG. 5. (Color online) Numerical density profile vs time $t$ (a) for $\left|u_{1}\right|^{2}$ and (b) for $\left|u_{2}\right|^{2}$. (c) Numerical (Num) and variational (Var) atom transfer ratio $R(t)$ vs time $t$. The parameters are $\Gamma=1, \phi(0)=$ $0, g=g_{12}=\gamma=0$, and $N_{2}(0)=0.5, N_{1}(0)=1, R(0)=\left[N_{2}(0)-\right.$ $\left.N_{1}(0)\right] /\left[N_{2}(0)+N_{1}(0)\right]=-0.3333$.

Next, we study the atom transfer between components for $g=g_{12} \neq 0$. In Fig. 6(a) we plot numerical and variational results for the atom transfer ratio $R(t)$ in several cases for $\gamma=$ 0 . The numerical results are in good agreement with variational equations (27) and (28), which shows that the period of $R(t)$ is related to only $\Gamma$, and the effect of $\phi(0)$ on $A$ and $B$ is larger than the effect of $R(0)$. Further investigations show that, if $\gamma \neq 0$, the density profiles may be non-Gaussian, and the variational equations (13)-(14) are no longer valid, although the atom transfer between two components can take place as demonstrated by lines $R 1$ and $R 2$ in Fig. 6(b). The plots in Fig. 6(b) indicate that the amplitude of $R$ changes periodically.
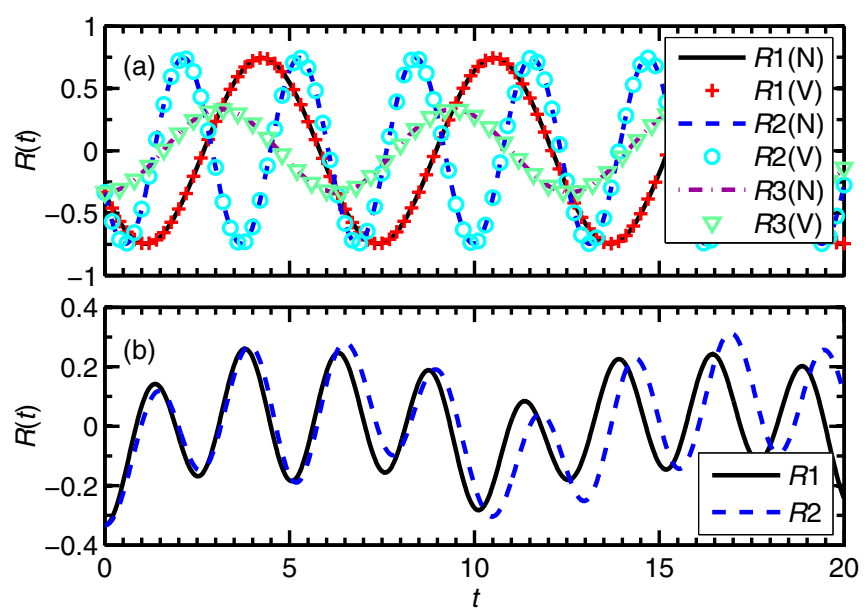

FIG. 6. (Color online) (a) Numerical (N) and variational (V) atom transfer ratio $R(t)$ vs time $t$ for $\gamma=0$ in different cases: $R 1$ is for $g=g_{12}=0, \phi(0)=\pi / 4, \Gamma=0.5 ; R 2$ is for $g=g_{12}=$ $-1, \phi(0)=\pi / 4, \Gamma=1$; and $R 3$ is for $g=g_{12}=-0.5, \phi(0)=$ $0, \Gamma=0.5$. (b) Numerical atom transfer ratio $R(t)$ vs time $t$ with $\phi(0)=0$ in different cases: $R 1$ is for $g=g_{12}=0, \Gamma=\gamma=1 ; R 2$ is for $g=0, g_{12}=-1, \Gamma=\gamma=1$. In all cases $N_{2}(0)=0.5, N_{1}(0)=$ $1, R(0)=\left[N_{2}(0)-N_{1}(0)\right] /\left[N_{2}(0)+N_{1}(0)\right]=-0.3333$. 


\section{SUMMARY}

Using the numerical solution and variational approximation of the time-dependent coupled mean-field GP equations with two pseudospin components, we studied the localization of the noninteracting and weakly interacting Bose-Einstein condensates with SO and Rabi couplings loaded in the quasiperiodic bichromatic OL potential (5). We use the set of binary GP equations (4) that accurately predicts the evolution of the atom transfer ratio $R(t)$, phase difference $\phi(t)$, and width $w$. The variational results leading to many physical insights are compared with the numerical results of the mean-field model. Stationary localized states of the model correspond to having the same number of atoms $\left(N_{1}=N_{2}\right)$ in two components. Nonstationary localized states with periodic atom transfer between components can be achieved for different numbers of atoms $\left(N_{1} \neq N_{2}\right)$. In the case of $\gamma \times \Gamma=0$, the density profiles of the two stationary localized states are symmetrical and are not related to the phase difference $\phi$, SO coupling $\gamma$, and Rabi coupling $\Gamma$. In the case of $\gamma \times \Gamma \neq 0$, the width of the stationary state should depend on the phase difference and the SO and Rabi couplings because of the interaction between $\gamma$ and $\Gamma$. It is found that the interaction between the SO coupling and Rabi coupling may favor a localization or delocalization depending on the phase difference between the two localized states. If $g=g_{12}$, a linear stability analysis shows that any stationary state is stable. We find that the BEC localized states always have a long exponential tail. In the case of $\phi=\pi$, the localization effect of a positive $\Gamma$ has a major influence on the localization length. We also studied some dynamics of the localized states with an atomic population imbalance and find $\Gamma$ and the initial phase difference play an important role in the atom transfer. Either in view of understanding the dynamic evolution or in view of the practical application, these properties are important. We hope that the present work will motivate new studies, especially experimental ones on the localization of BEC with the SO coupling.

\section{ACKNOWLEDGMENTS}

FAPESP and CNPq (Brazil) provided partial support. Y.C. is supported by National Natural Science Foundation of China Grant No. 11274104, Provincial Natural Science Foundation of Hubei Grant No. 2011CDA021, and the Science and Technology Program of the Education Department of Hubei Grant No. Q20132507.
[1] Y.-J. Lin, K. Jiménez-García, and I. B. Spielman, Nature (London) 471, 83 (2011).

[2] V. Galitski and I. B. Spielman, Nature (London) 494, 49 (2013).

[3] J. Higbie and D. M. Stamper-Kurn, Phys. Rev. Lett. 88, 090401 (2002); T. L. Ho and S. Zhang, ibid. 107, 150403 (2011); Y. Deng, J. Cheng, H. Jing, C. P. Sun, and S. Yi, ibid. 108, 125301 (2012); J. Radic, T. A. Sedrakyan, I. B. Spielman, and V. Galitski, Phys. Rev. A 84, 063604 (2011).

[4] P. Wang, Z.-Q. Yu, Z. Fu, J. Miao, L. Huang, S. Chai, H. Zhai, and J. Zhang, Phys. Rev. Lett. 109, 095301 (2012); L. W. Cheuk, A. T. Sommer, Z. Hadzibabic, T. Yefsah, W. S. Bakr, and M. W. Zwierlein, ibid. 109, 095302 (2012).

[5] J.-Y. Zhang, S.-C. Ji, Z. Chen, L. Zhang, Z.-D. Du, Bo Yan, G.-S. Pan, Bo Zhao, Y.-J. Deng, H. Zhai, S. Chen, and J.-W. Pan, Phys. Rev. Lett. 109, 115301 (2012); M. Aidelsburger, M. Atala, S. Nascimbène, S. Trotzky, Y.-A. Chen, and I. Bloch, ibid. 107, 255301 (2011); Z. Fu, P. Wang, S. Chai, L. Huang, and J. Zhang, Phys. Rev. A 84, 043609 (2011); C. Qu, C. Hamner, M. Gong, C. Zhang, and P. Engels, ibid. 88, 021604 (2013).

[6] D. W. Zhang, J. P. Chen, C. J. Shan, Z. D. Wang, and S. L. Zhu, Phys. Rev. A 88, 013612 (2013); Q. Zhu, C. Zhang, and B. Wu, Europhys. Lett. 100, 50003 (2012).

[7] X. F. Zhou, J. Zhou, and C. Wu, Phys. Rev. A 84, 063624 (2011).

[8] Y. Xu, Y. Zhang, and B. Wu, Phys. Rev. A 87, 013614 (2013); O. Fialko, J. Brand, and U. Zülicke, ibid. 85, 051605(R) (2012).

[9] M. Gong, G. Chen, S. Jia, and C. Zhang, Phys. Rev. Lett. 109, 105302 (2012); M. Gong, S. Tewari, and C. Zhang, ibid. 107, 195303 (2011); H. Hu, L. Jiang, X. J. Liu, and H. Pu, ibid. 107, 195304 (2011); L. Han and C. A. R. Sáde Melo, Phys. Rev. A 85, 011606 (2012).
[10] Y. Zhang, L. Mao, and C. Zhang, Phys. Rev. Lett. 108, 035302 (2012).

[11] T. D. Stanescu, B. Anderson, and V. Galitski, Phys. Rev. A 78, 023616 (2008); J. Larson and E. Sjöqvist, ibid. 79, 043627 (2009).

[12] L. Salasnich and B. A. Malomed, Phys. Rev. A 87, 063625 (2013).

[13] Y. Li, G. I. Martone, L. P. Pitaevskii, and S. Stringari, Phys. Rev. Lett. 110, 235302 (2013); Y. Zhang and C. Zhang, Phys. Rev. A 87, 023611 (2013).

[14] C. Wang, C. Gao, C. M. Jian, and H. Zhai, Phys. Rev. Lett. 105, 160403 (2010); T. Kawakami, T. Mizushima, and K. Machida, Phys. Rev. A 84, 011607(R) (2011); A. Aftalion and P. Mason, ibid. 88, 023610 (2013).

[15] P. W. Anderson, Phys. Rev. 109, 1492 (1958).

[16] J. Billy, V. Josse, Z. Zuo, A. Bernard, B. Hambrecht, P. Lugan, D. Clément, L. Sanchez-Palencia, P. Bouyer, and A. Aspect, Nature (London) 453, 891 (2008).

[17] G. Roati, C. D'Errico, L. Fallani, M. Fattori, C. Fort, M. Zaccanti, G. Modugno, M. Modugno, and M. Inguscio, Nature (London) 453, 895 (2008).

[18] L. Fallani, J. E. Lye, V. Guarrera, C. Fort, and M. Inguscio, Phys. Rev. Lett. 98, 130404 (2007).

[19] S. S. Kondov, W. R. McGehee, J. J. Zirbel, and B. DeMarco, Science 334, 66 (2011).

[20] F. Jendrzejewski, A. Bernard, K. Müller, P. Cheinet, V. Josse, M. Piraud, L. Pezzé, L. Sanchez-Palencia, A. Aspect, and P. Bouyer, Nat. Phys. 8, 398 (2012).

[21] B. Damski, J. Zakrzewski, L. Santos, P. Zoller, and M. Lewenstein, Phys. Rev. Lett. 91, 080403 (2003); L. Sanchez-Palencia, D. Clément, P. Lugan, P. Bouyer, G. V. Shlyapnikov, and A. 
Aspect, ibid. 98, 210401 (2007); S. E. Skipetrov, A. Minguzzi, B. A. van Tiggelen, and B. Shapiro, ibid. 100, 165301 (2008); A. Yedjour and B. A. Van Tiggelen, Eur. Phys. J. D 59, 249 (2010); M. Piraud, L. Pezze, and L. Sanchez-Palencia, Europhys. Lett. 99, 50003 (2012); New J. Phys. 15, 075007 (2013); J. Biddle, B. Wang, D. J. Priour, and S. Das Sarma, Phys. Rev. A 80, 021603 (2009); M. Larcher, F. Dalfovo, and M. Modugno, ibid. 80, 053606 (2009); M. Modugno, New J. Phys. 11, 033023 (2009); Y. Cheng and S. K. Adhikari, Phys. Rev. A 83, 023620 (2011); 84, 023632 (2011); 84, 053634 (2011); 82, 013631 (2010); S. K. Adhikari and L. Salasnich, ibid. 80, 023606 (2009); M. Takahashi, H. Katsura, M. Kohmoto, and T. Koma, New J. Phys. 14, 113012 (2012).

[22] L. Zhou, H. Pu, and W. Zhang, Phys. Rev. A 87, 023625 (2013).

[23] M. J. Edmonds, J. Otterbach, R. G. Unanyan, M. Fleischhauer, M. Titov, and P. Öhberg, New J. Phys. 14, 073056 (2012).

[24] V. M. Pérez-García, H. Michinel, J. I. Cirac, M. Lewenstein, and P. Zoller, Phys. Rev. A 56, 1424 (1997); Y. Cheng, R. Z. Gong, and H. Li, Opt. Express 14, 3594 (2006); B. A. Malomed, Prog. Opt. 43, 71 (2002).

[25] P. Bouyer, Rep. Prog. Phys. 73, 062401 (2010); L. SanchezPalencia and M. Lewenstein, Nat. Phys. 6, 87 (2010); L. Fallani,
C. Fort, and M. Inguscio, Adv. At. Mol. Opt. Phys. 56, 119 (2008)

[26] Y. A. Bychkov and E. I. Rashba, J. Phys. C 17, 6039 (1984).

[27] G. Dresselhaus, Phys. Rev. 100, 580 (1955).

[28] L. Salasnich, A. Parola, and L. Reatto, Phys. Rev. A 65, 043614 (2002); C. A. G. Buitrago and S. K. Adhikari, J. Phys. B 42, 215306 (2009).

[29] S. Blatt, T. L. Nicholson, B. J. Bloom, J. R. Williams, J. W. Thomsen, P. S. Julienne, and J. Ye, Phys. Rev. Lett. 107, 073202 (2011).

[30] S. L. Cornish, N. R. Claussen, J. L. Roberts, E. A. Cornell, and C. E. Wieman, Phys. Rev. Lett. 85, 1795 (2000); M. Theis, G. Thalhammer, K. Winkler, M. Hellwig, G. Ruff, R. Grimm, and J. H. Denschlag, ibid. 93, 123001 (2004); S. E. Pollack, D. Dries, M. Junker, Y. P. Chen, T. A. Corcovilos, and R. G. Hulet, ibid. 102, 090402 (2009); S. Inouye, M. R. Andrews, J. Stenger et al., Nature (London) 392, 151 (1998).

[31] N. F. Mott, J. Non-Cryst. Solids 1, 1 (1968).

[32] S. Flach, D. O. Krimer, and Ch. Skokos, Phys. Rev. Lett. 102, 024101 (2009); A. S. Pikovsky and D. L. Shepelyansky, ibid. 100, 094101 (2008). 